\title{
LA LOGÍSTICA DE REVERSA COMO ESTRATEGIA COMPLEMENTARIA EN LA PRODUCCIÓN MÁS LIMPIA Y EL DESARROLLO HUMANO SOSTENIBLE
}

\section{Reverse Logistics as a Complementary Strategy in Cleaner Production and Sustainable Human Development}

\author{
Claudia Peña-Montoya ${ }^{1}$, Patricia Torres-Lozada²
}

${ }^{1}$ Ingeniera Industrial M.Sc, Estudiante doctorado en Ingeniería, Universidad del Valle.

2Ingeniera Sanitaria, Ph.D, Profesora titular, EIDENAR, Universidad del Valle

E-mail: claudia.pena@correounivalle.edu.co

Recibido: 3 de Junio de 2013

Aceptado: 5 de Septiembre de 2013

\section{Resumen}

La gestión ambiental en pequeñas y medianas empresas -PyMEs aún carece del reconocimiento de los beneficios que ésta puede generar en aspectos como la competitividad y la productividad. Para maximizar los beneficios es necesario explorar el efecto positivo de integrar estrategias como la producción más limpia, la logística de reversa y el desarrollo humano sostenible en aspectos orientados a la gestión de residuos sólidos. En este artículo se evalúa la situación actual de una PyME de plásticos respecto a la integración de las tres estrategias mencionadas. Los resultados mostraron que si bien se tienen fortalezas en aspectos productivos y organizacionales, aún es prioritario atender aspectos ambientales y del bienestar humano en torno a la gestión de residuos sólidos.

Palabras claves: Desarrollo humano sostenible, Logística de reversa, Producción más limpia, PyMEs, Rosa de los vientos.

\section{Abstract}

Small and medium size enterprises- SMEs require of acknowledgment for the benefits from environmental management on competitive and productivity aspects. It is necessary to explore the positive effects obtained from the integration of strategies such as cleaner production, reverse logistics and sustainable human development on solid waste management. This work is aimed at evaluating the current situation of a SME in the plastic sector regarding the three strategies above mentioned. Results showed that the strengths of the company are in the productive and organizational aspects while environmental and welfare aspects need further attention regarding solid waste management.

Keywords: Sustainable human development, Reverse logistics, Cleaner production, SMEs, wind rose. 


\section{INTRODUCCIÓN}

Durante las dos últimas décadas, los aspectos ambientales de la gestión de la cadena de suministro han tomado gran auge entre académicos y empresarios, debido principalmente a las regulaciones impuestas por gobiernos de varios países y a que los empresarios han explorado estrategias de reducción de costos a través de la recuperación de materiales (Golinska \& Romano 2012). Sin embargo, es necesario que las estrategias que mejoran el desempeño ambiental de las cadenas de suministro actúen de manera complementaria, para maximizar los beneficios de la integración.

Los esfuerzos para mejorar el desempeño de las cadenas de suministro a través de la gestión ambiental han conducido a mejorar los sistemas productivos, lo que se refleja en la calidad de productos y procesos y la flexibilidad de la organización al integrarse con proveedores, distribuidores y consumidores. Entre las estrategias de gestión ambiental, se han adoptado los principios de producción más limpia (PML) en el área de producción de las empresas, pasando de soluciones a final de tubo hacia acciones preventivas desde los primeros eslabones de la cadena de valor, fundamentadas principalmente en la reducción en la generación de los residuos sólidos y en el aprovechamiento y valorización de los generados.

Para abordar aspectos específicos de la gestión ambiental como la gestión de residuos sólidos, se dispone de la estrategia de logística de reversa (LR), que permite aprovechar y valorizar productos en algún punto de la cadena de valor, evitándose el consumo acelerado de materia primas vírgenes y el incremento de residuos sólidos. Sin embargo, la implementación de estrategias de gestión ambiental requiere de fortalezas financieras $y$ organizacionales, lo cual representa una restricción para empresas pequeñas y medianas (PyMEs) cuyo presupuesto es reducido y se orienta principalmente a atender aspectos prioritarios de las operaciones como son suministro de materiales, disponibilidad de maquinaria y mano de obra (Becerra \& Van Hoof 2004).

Por otro lado, según Fajardo (2006), el Desarrollo Humano Sostenible (DHS) se considera una forma de desarrollo pero centrada en el ser humano, tanto a nivel individual como a nivel colectivo, fortaleciendo las capacidades comunitarias, generando mayores niveles de bienestar social $y$ disminuyendo los niveles de pobreza. Además, el DHS se ocupa del control demográfico, la distribución equitativa de los recursos, acceso a servicios de salud, la participación en gobiernos cada vez más descentralizados, el comercio equitativo y abierto (Santamaría 1997).

En este documento se analiza la logística de reversa como estrategia complementaria para la producción más limpia y el desarrollo humano sostenible en una PyME del sector de plásticos del Valle del Cauca, Colombia. Además, se plantea la necesidad de reconocer el principio de la complementariedad en la gestión ambiental empresarial en PyMEs, las cuales son prioritarias en Colombia porque generan más del 50\% del empleo nacional, representan el 36\% del valor agregado industrial, el $92 \%$ de los establecimientos comerciales y $40 \%$ de la producción total del país (Concha 2011).

\section{ANTECEDENTES}

\section{Producción Más Limpia}

De acuerdo son el PNUMA (2012), la PML es la aplicación continua de una estrategia ambiental integral y preventiva a los procesos, productos y servicios para incrementar la eficiencia y reducir los efectos negativos al ambiente y la salud humana. Si se tiene en cuenta que la definición considera la prevención, entonces debería ser común encontrar que las estrategias asociadas con la PML, anticiparán los efectos de acciones de los sistemas productivos como el excesivo consumo de recursos naturales, la dependencia tecnológica y el exceso de residuos y emisiones, de tal modo que no se tomen medidas después que éstos ocurren, a lo que comúnmente se llama soluciones de final de tubo. Soluciones como el tratamiento de residuos y emisiones, se reconocen que no son apropiadas para la integralidad de la gestión ambiental (Zeng et al. 2008); por lo tanto, las empresas han venido adoptando acciones que modifiquen la orientación de la PML hacia una estrategia integral de producción, consumo, comercio y desarrollo.

Esta nueva orientación de la PML debe considerar el análisis cuidadoso de los proveedores de las materias primas, diseño de los productos, procesos de producción, transporte, uso y disposición final, ya que inicialmente se consideraban de manera aislada (Van Hoof \& Herrera 2007). Por lo tanto, al optimizar los diseños de los productos, se abren las posibilidades de mejoramiento en todas las demás etapas del ciclo de vida, incluyendo los procesos productivos, su manera de distribuirlos y disponerlos.

Según Herrera (2009), la gestión ambiental empresarial en Colombia ha presentado una evolución de acuerdo con los lineamientos de la Política de Producción más Limpia, donde antes se concentraban esfuerzos en torno a la gestión de residuos y emisiones atmosféricas, mientras que actualmente el objetivo es evitar la producción de residuos y disminuir el uso de materias primas y energía. Sin embargo, la incorporación de prácticas de PML se ha centrado en la grandes empresas, mientras que su impulso en las PyMEs 
apenas se está promoviendo con el apoyo de los Centros Regionales de PML (Van Hoof \& Herrera 2007).

Específicamente en el manejo de residuos sólidos a nivel empresarial, se han adelantado esfuerzos para gestionarlos al final del ciclo de vida, principalmente los residuos peligrosos, lo cual se ha ampliado a otros residuos. No obstante, con la aparición de la Política de Producción y Consumo Sostenible (Colombia 2010) la orientación se ha concentrado en la responsabilidad de fabricantes o importadores de cumplir metas y plazos de recuperación de materiales; así mismo se plantea la responsabilidad en el consumo de los ciudadanos porque según la Política no sólo los procesos serán más limpios sino también los productos. Aquí los ciudadanos se ven reflejados en los actores sociales como la academia, la industria y el gobierno; además, a nivel de industria se requiere de la participación de todas las áreas funcionales y colaboración entre empresas.

\section{Gestión de las Cadenas de Suministro y Logística de Reversa}

De acuerdo con Ballou (2004) es difícil separar la definición de gestión de logística de la gestión de la cadena de suministro por cuanto las dos se orientan a la misma misión: suministrar los bienes o servicios en el lugar preciso, en el momento indicado y en las condiciones deseadas, al tiempo que se genera valor para el negocio. Por lo tanto, Ballou (2004) reconoció la integración de los dos términos para definir que logística o gestión de la cadena de suministro es la integración de actividades funcionales (transporte, control de inventarios, distribución) que se repiten varias veces a través del canal en el cual la materia prima se convierte en producto terminado y se agrega valor al consumidor final.

Las cadenas de suministro como estructuras operacionales que gestionan materias primas y entregan productos al consumidor final (Ballou 2004) están involucradas en la gestión de residuos sólidos, representados en excedentes de manufactura, disminución de valor de materiales y productos que llegan al final de su ciclo de vida. Para gestionar estos residuos de una manera adecuada, existe la estrategia de LR que se ocupa de planear, implementar y controlar el flujo eficiente y costo efectivo de materias primas, producto en proceso, producto terminado, empaques, devoluciones desde el punto de consumo hasta un punto de recuperación o disposición adecuada (Rogers \& Tibben-Lembke 1998). El punto de consumo se refiere al origen de los materiales que pueden ser devoluciones de manufactura resultado de control de calidad o sobrantes de producción, devoluciones de distribución por ajustes de inventarios, deterioro de embalaje y devoluciones de los clientes por garantías o final del ciclo de vida (De Brito \& Dekker 2004).
La LR es un componente de la gestión de la cadena de suministro a través de la cual es posible la incorporación en el ciclo productivo de materiales o productos que han perdido o disminuido su valor, como factor que cobra mayor importancia entre los aspectos ambientales que se incluyen en la estrategia corporativa, porque suponen una fuente de ventaja competitiva al reducir costos económicos y ambientales (Díaz et al. 2004).

La aplicación de la LR en Colombia se aprecia a nivel de grandes empresas (Monroy \& Ahumada 2006), mientras que los estudios que presenten el efecto de la aplicación de esta estrategia en PyMEs son escasos, lo cual tiende a asemejarse con las prácticas de PML que apoyan de los centros regionales de PML. En general, las experiencias de LR en Colombia resaltan la implementación de la estrategia para alcanzar la competitividad de las grandes empresas privilegiando la orientación económica sobre la ambiental porque se recupera el valor de los productos devueltos (Monroy \& Ahumada 2006, Cure et al. 2006, Mihi 2007).

\section{Producción más Limpia y Desarrollo Humano Sostenible}

Al reconocer la especificidad del hombre y su papel como parte integral de la naturaleza, se puede analizar la actual problemática ambiental como un espacio de interacción entre el mundo natural y el mundo cultural (Pérez \& Rojas 2010), en el cual es posible contextualizar el DHS que considera al ser humano como parte de la naturaleza formando un todo indivisible. Por lo anterior, es importante reconocer la responsabilidad que el ser humano tiene en su relación hombre-naturaleza, porque dado el desarrollo tecnológico actual, el hombre pretende subyugar la naturaleza poniéndola a su servicio (De Sequeira 2009), convirtiéndose en responsable de hechos tan conocidos como el cambio climático.

Por otra parte, la PML, como estrategia orientada a garantizar la sostenibilidad de los procesos productivos y el uso eficiente de recursos como agua y energía, se vincula con el DHS al reconocer e integrar las habilidades, competencias y necesidades de las comunidades involucradas en el ciclo de producción y consumo.

Aquí es preciso introducir el concepto de complementariedad, que considera que dos redes de conceptos aunque mutuamente excluyentes se integran para conocer acerca de un mismo objeto (Roldán et al. 2004) y así plantear que el efecto de la LR, PML y el DHS sobre las cadenas productivas es más ventajoso al considerarlas integradamente y no de forma aislada. Además, el sistema productivo se considera no lineal y complejo lo que corresponde a la sostenibilidad fuerte; de éste modo el 
desarrollo económico debe hacerse compatible con la dinámica de crecimiento y sostenibilidad de los bienes naturales y el desarrollo humano (Pérez \& Rojas, 2010).

Así mismo, se podría afirmar que el paradigma de la complementariedad está presente por ejemplo, en el nombre mismo de la estrategia de LR, Logística (técnica) de Reversa (reincorporación al ciclo productivo para reducir consumo de recursos y aumentar los efectos positivos al ambiente). De ahí que buena parte de la literatura de LR se refiera a aspectos como medio ambiente y competitividad (Díaz et al. 2004, Leite 2010) en un intento por migrar del paradigma de producciónconsumo hacia el de producción- calidad de vida; sin embargo, se evidencia la necesidad de integrar los aspectos del DHS para comprender que sólo es posible alcanzar competitividad y óptimo desempeño ambiental sí se consideran articuladamente la interacción entre sociedad, cultura, ambiente y productividad.

Lo anterior es un reto que se impone dada la falta de integración holística de la cadena de suministro con la sostenibilidad, porque según De Brito \& Van Der Laan (2010) las cadenas de suministro se ocupan especialmente de asuntos inherentes a la gestión de operaciones y muy poco a asuntos sociales o ambientales. Sin duda, lo anterior constituye un llamado a trabajar interdisciplinariamente en la gestión de la cadena de suministro, de tal modo que la LR consolide vínculos con asuntos ecológicos, tecnológicos, legales, sociales y económicos (De Brito 2003).

Algunos avances en la concepción holística de la gestión de operaciones se dan al reconocer que el éxito de los programas de PML radica en la implementación de programas de LR exitosos que involucren la recolección, selección, procesamiento, transporte y almacenamiento que reciclen los residuos sólidos de la planta de producción o los subproductos, regresándolos al lugar de origen o a nuevos consumidores (Martin 2001).

\section{CONTEXTO EMPRESARIAL}

\section{Caso estudio, Empresa del sector plástico del Valle del Cauca- Colombia}

La empresa bajo estudio, es una empresa mediana localizada departamento del Valle del Cauca, Colombia que se dedica a la fabricación de productos plásticos inyectados y a la elaboración de moldes para inyección, cuyos clientes nacionales e internacionales incluyen pequeñas empresas y multinacionales. El proceso de inyección es aquel donde se generan mayor cantidad de residuos plásticos, los cuales se muelen para pasar a tamaños pequeños en el proceso de remolido y regresan al proceso productivo según se aprecia en la Figura 1.

Las personas encargadas de la toma de decisiones en la empresa, son conscientes que al aprovechar éste material están disminuyendo el consumo de materia prima virgen y también están minimizando la cantidad de residuos sólidos aprovechables que irían a disposición final, aumentando así la vida útil de estos sitios, disminuyendo la generación de gases de efecto invernadero y la producción de lixiviados; no obstante, no se valora económica, ambiental y socialmente los beneficios de ésta práctica para la empresa.

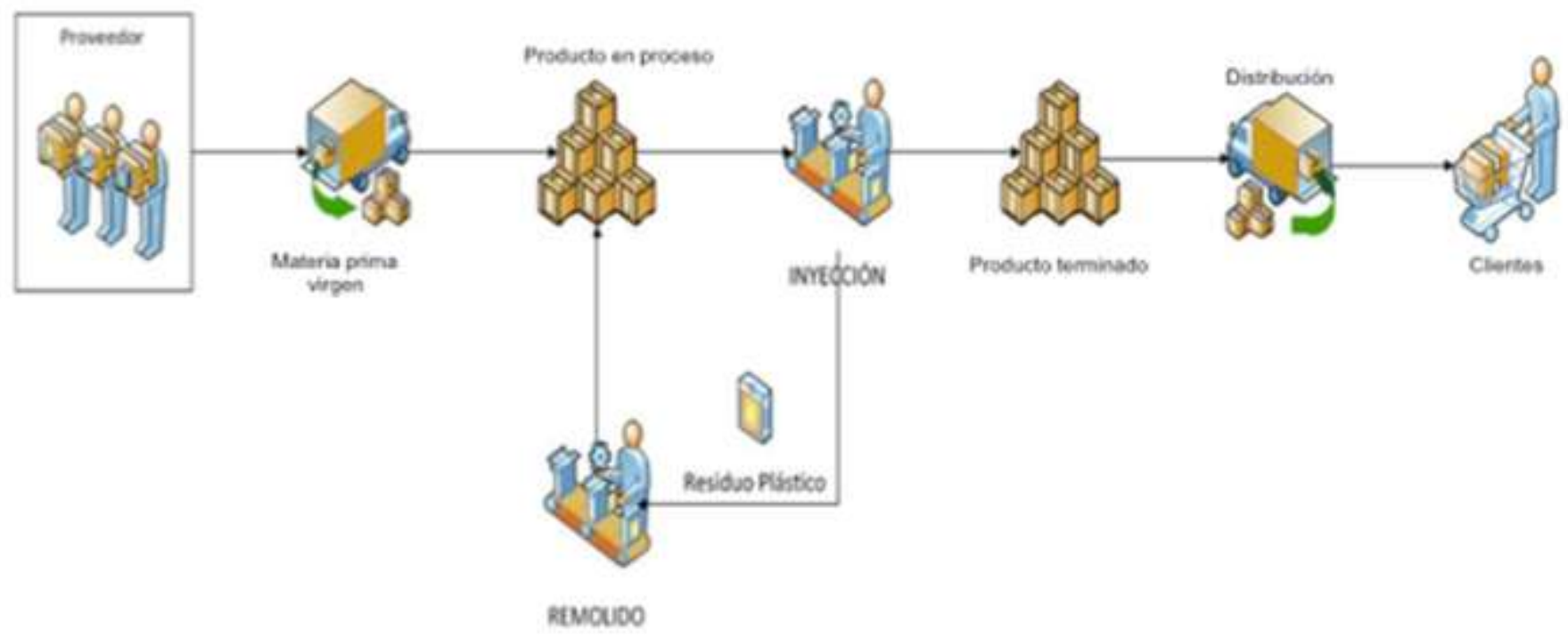

Figura 1. Recuperación de los residuos del proceso productivo 
Ambiente y Sostenibilidad 2013 (1): 7-14

Revista del Doctorado Interinstitucional en Ciencias Ambientales

ISSN: 2339-3122

\section{METODOLOGÍA}

Teniendo en cuenta que la PML es una estrategia lineal que se concentra en la optimización de procesos y productos para minimizar los residuos o subproductos descargados al ambiente, es necesario incluir una visión complementaria que involucre otras áreas funcionales de la empresa y de alguna manera considere la influencia de las operaciones en el entorno en que se desenvuelve tales como el complejo industrial, los gremios y la comunidad. Se reconoce la necesidad de integración de los principios ambientales basados en la complementariedad (Roldán et al. 2004) con los criterios orientadores de la LR, PML y el DHS, pues se debe salir de los límites de la empresa para considerar cómo los productos, las prácticas productivas y las relaciones de negocios afectan la comunidad, los empleados y sus familias, los ecosistemas y las relaciones entre países.

Por lo anterior, se usó la metodología de la rosa de los vientos ampliamente empleada en procesos de evaluación de desempeño académico, municipal y agrícola en la cual se integran elementos que parecían excluyentes para comprender el beneficio de la interacciones. Para este caso, se consideraron factores de producción, organizacionales, de bienestar y ambientales porque para ellos explican en gran medida la relación entre LR, PML y el DHS, ofreciendo una visión holística de la empresa en torno la recuperación de materiales. Para cada factor se definieron cuatro características y a cada característica se asoció una pregunta. Las preguntas formuladas se exponen a continuación:

\section{Producción}

1. ¿Es la infraestructura y tecnología adecuada para reducir defectos de calidad, desperdicios y reprocesarlos?

2. ¿Tienen implementados y hacen seguimiento a sistemas de gestión de calidad?

3. ¿Se consideran opciones de gestión para los subproductos y residuos que resultan del proceso productivo?

4. ¿En qué medida contribuye las actividades de reprocesamiento en la rentabilidad de la empresa?

\section{Organizacional}

1. ¿Es la estructura administrativa flexible y respalda la competitividad de la empresa?

2. ¿En qué medida los procesos administrativos impactan la rentabilidad de la empresa?

3. Califique la capacidad gerencia y gestión para administrar y asignar efectivamente los recursos

4. ¿Cuál es el grado de adaptación a los cambios que imponen la competencia, tratados de libre comercio y de sostenibilidad?
Bienestar

1. ¿Se maneja buenas prácticas de manufactura y seguridad industrial?

2. ¿Cómo se manejan aspectos como estabilidad laboral, remuneración, seguridad social y turnos de trabajo?

3. ¿Se tienen en cuenta aspectos como enfoque de género y familia con los empleados?

4. ¿Existen oportunidades de carrera dentro de la compañía?

\section{Ambiental}

1. ¿La tecnología empleada influye en la reducción de las emisiones atmosféricas?

2. ¿Se gestionan adecuadamente los residuos peligrosos?

3. ¿Qué avances presenta la empresa en gestión ambiental, tiene implementado la norma ISO 14000 por ejemplo?

4. ¿Existen planes de aprovechamiento y valorización de residuos sólidos?

Se preguntó a los gerentes de las áreas administrativas y de operaciones de la empresa para dar lugar a la ponderación (respuesta de los gerentes, escala 1-10) y se valoró la situación actual respecto a las preguntas de acuerdo con la observación in situ del investigador, para así obtener el grado de cumplimiento (escala 1-5) de cada característica; el producto de ambos se denominó evaluación, es decir la integración de las respuestas obtenidas. El logro ideal supone que el grado de cumplimiento toma la máxima escala de 5 y se multiplica por la ponderación aportada por los gerentes; la división de la evaluación y el logro ideal da lugar a la relación final, la cual aporta los datos para graficar la denominada rosa de los vientos. El área de la rosa de los vientos ideal, es decir cuando todos los valores alcanzan la máxima calificación, se divide entre el área de la rosa de los vientos actual (situación actual de la empresa) para obtener el Índice de Sostenibilidad de Producción más Limpia- ISPML.

Si la calificación real a todas las categorías alcanzara la máxima puntuación, el ISPML sería 1,0; por lo tanto valores menores demuestran que los factores evaluados tienen aspectos por mejorar. Esta herramienta permite además hacer seguimiento a los aspectos identificados e integrar otros que necesiten ser evaluados.

\section{RESULTADOS}

La Tabla 1 presenta un resumen de las respuestas de los gerentes y la evaluación del investigador en cada factor frente a la situación actual de la empresa. Por ejemplo en el factor producción se aprecia que para la característica de infraestructura y tecnología cuya pregunta es la número uno 
en la sección de preguntas, los gerentes le dieron una ponderación de 10 (escala 1-10) y el investigador de 5 (escala 1-5), para obtener una relación final de 1,0.

Tabla 1. Valoraciones de la situación actual de la empresa

\begin{tabular}{|c|c|c|c|c|c|c|}
\hline Factor & Característica & $\begin{array}{c}\text { (a) } \\
\text { Ponderación }\end{array}$ & $\begin{array}{l}\text { (b) Grado de } \\
\text { Cumplimiento }\end{array}$ & $\begin{array}{c}\left(a^{*} b\right)=c \\
\text { Evaluación }\end{array}$ & $\begin{array}{c}\left(5^{*} \mathrm{a}\right)=\mathrm{d} \\
\text { Logro Ideal }\end{array}$ & $\begin{array}{c}\text { (c/d) Relación } \\
\text { Final }\end{array}$ \\
\hline Producción & Infraestructura y Tecnología & 10 & 5 & 50 & 50 & 1.00 \\
\hline Organizacional & Procesos Administrativos & 8 & 4 & 32 & 40 & 0.80 \\
\hline Bienestar & Seguridad Industrial & 9 & 5 & 45 & 45 & 1.00 \\
\hline Ambiental & Aprovechamiento y Valoración & 7 & 3 & 21 & 35 & 0.60 \\
\hline
\end{tabular}

A partir de la información arrojada por la relación final, se graficó la rosa de los vientos (Figura 2), la cual indica que la empresa presenta punto críticos y potencialidades en la relación de LR, PML y el DHS. En esta Figura los primeros cuatro resultados corresponden al factor de producción, seguidos por el organizacional, bienestar y ambiental (cuatro resultados por factor).

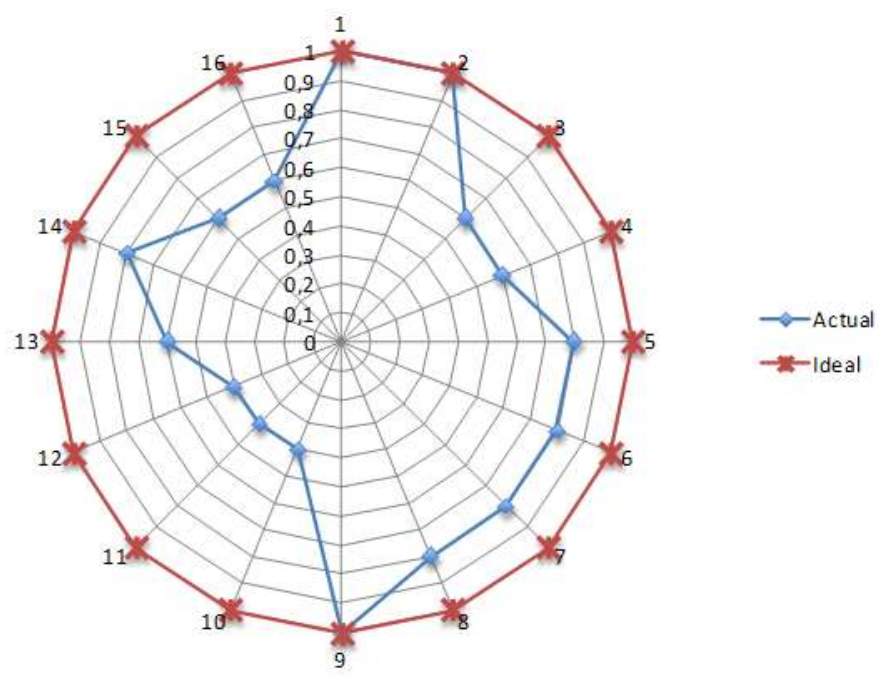

Figura 2. Rosa de los vientos para la empresa del estudio

Los aspectos productivos (1-4) se comportaron de manera aceptable de acuerdo con la valoración obtenida, lo cual se justifica porque existe la tecnología adecuada para los procesos productivos y para reprocesar los subproductos que resultan de los mismos; sin embargo, aún las PyMEs enfrentan debilidades como baja rentabilidad de sus operaciones y escasa incorporación de tecnología (Silva 2011). Las potencialidades se presentan de manera sostenida en los factores organizacionales (5-8) en los cuales la estructura de la empresa apoya las actividades de recuperación de materiales, gestiona los recursos necesarios para la operación de la empresa eficientemente $\mathrm{y}$ es flexible para adaptarse a los cambios que imponen la legislación, la competencia y la sociedad.
Los puntos críticos se evidencian en el factor de bienestar del personal que labora en la empresa (9-12), porque aspectos como estabilidad laboral, remuneración, seguridad social, turnos de trabajo, enfoque de género, familia y oportunidades de carrera presentan debilidades que la empresa debe fortalecer a través del monitoreo de acciones orientadas a la integración de la PML, el DHS y la LR. Se destaca la marcada preferencia por vincular personal masculino a nivel de planta de producción que, según indagaciones, está fundamentada en mayores capacidades cognitivas y conocimientos básicos en procesos con plásticos que se adquieren a través de entidades de formación como el SENA. Este hecho ofrece un espacio para consolidar los principios del DHS con las otras dos estrategias, a la luz del aspecto social presente en la sostenibilidad.

Los aspectos ambientales (13-16) se consideran aceptables, identificándose la falta de implementación de la norma ISO 14000 y de programas formales de aprovechamiento y valorización de residuos sólidos que resultan del proceso productivo o de programas postconsumo. El motivo de esta última coincide con lo señalado por da Silva \& Moita Neto (2011) porque los costos que deben enfrentar los empresarios para aprovechar materiales a través de la LR, pueden ser más altos que adquirir materia prima virgen. Además, para las PyMEs no es clara la relación positiva entre gestión ambiental y competitividad social y económica (Becerra \& Van Hoof 2004) y por el contrario, le asocian incremento de costos y reducción de la rentabilidad.

Adicionalmente, los tomadores de decisiones de la empresa argumentan que sólo involucran una pequeña proporción de resina postproducción que proviene de procesos internos y de otras empresas porque en Colombia predomina la contaminación del material postconsumo recuperado (Monroy \& Ahumada 2006).

Cabe anotar, que las opciones de gestión de residuos sólidos son escogidas con base en la experiencia de los tomadores de decisiones, lo cual es una deficiencia pues se podría obtener beneficios al adoptar los principios de la PML para formular el plan de manejo de residuos sólidos que mejore el 
desempeño ambiental, productividad y rentabilidad y cumplimiento de legislación actual (Muñoz \& Muñoz 2007).

Aún se no ha configurado un sistema de LR (recolección, selección, transporte, reprocesamiento y almacenamiento) al interior de la empresa, de modo que le permita usar efectivamente los recursos disponibles y cuantificar los beneficios potenciales, pues según lo observado, se presentan actividades de recuperación aisladas que no se fundamentan bajo los principios de PML o LR. Esto guarda relación con lo expuesto por Monroy \& Ahumada (2006) ya que a diferencia de las PyMES, las empresas grandes disponen de capital e infraestructura, así como de políticas de gestión ambiental basadas en el desarrollo sostenible; por lo tanto, es más común el desarrollo de estas estrategias.

Finalmente, a partir de la rosa de los vientos actual e ideal se obtuvo el ISPML de la empresa (relación actual/relación ideal) el cual fue del $50.2 \%$; este es un valor intermedio que se podría atribuir al desconocimiento de la complementariedad en el desempeño de la empresa y su entorno, por lo tanto se presenta una oportunidad para fortalecer la integralidad de los factores de manera que su seguimiento permita incrementar el ISPML con el trabajo interdisciplinario de representantes de la empresa, del sector y de la comunidad académica.

\section{CONCLUSIONES}

Con base en los resultados obtenidos se puede concluir que es necesaria la construcción y fortalecimiento del paradigma producción-calidad de vida de la sociedad (consumidores, trabajadores) en la gestión empresarial, principalmente en PyMEs cuyo capital y estructura organizacional es débil, para así contribuir significativamente al cumplimiento de los objetivos de la Política de Producción y Consumo Sostenible.

El análisis presentado aporta a la documentación de la situación de un PyME del sector plástico en la necesidad de integración de LR, PML y el DHS, usando la metodología de la rosa de los vientos. Sin embargo, se debe ampliar el estudio para considerar mayor número de empresas en el mismo sector $\mathrm{u}$ otros sectores $\mathrm{y}$ adicionalmente se puede focalizar por zonas industriales para maximizar los beneficios económicos, sociales y ambientales de aprovechar y valorizar materiales que son considerados residuos sólidos. Para maximizar los beneficios de estrategias complementarias como la LR, PML y el DHS, es necesario iniciar por el reconocimiento de las habilidades y competencias de los mismos colaboradores de la empresa en recuperar los materiales de forma planificada y organizada, de forma que sean ellos los primeros llamados a integrar las cooperativas o grupos de recuperadores de materiales que podrían actuar como proveedores de la misma empresa y de otras empresas del sector y de la zona industrial.

\section{REFERENCIAS}

BALLOU, R. H. 2004. Business Logistics/ Supply Chain Management, 5 ed. Pearson Prentice Hall, New Jersey. 816 pp.

BAUMAN, Z. 2007. Vida de consumo. Fondo de Cultura Económica, México. 208 pp.

BECERRA, M. \& VAN HOOF, B. 2004. Para que la Pyme sea más competitiva se requiere una estrategia ambiental preventiva. $\begin{array}{llllll}\text { Consultado el } 25 & \text { de }\end{array}$ http://www.manuelrodriguezbecerra.com/tmacolombia.htm

COLOMBIA 2010. Política Nacional de Producción y Consumo Sostenible. En: COLOMBIA, Ministerio de Ambiente, Vivienda y Desarrollo Territorial (ed.). Bogotá, DC.

CONCHA, J. R. 2011. Oportunidades de negocio para las Pymes Colombianas. En: Visión Mipyme Cali: ACOPI- Valle del Cauca.

CURE, L. MESA, J. C. \& AMAYA, M. 2006. Logística Inversa: Una herramienta de apoyo a la competitividad de las organizaciones. Ingeniería \& Desarrollo 20: 184-202.

DA SILVA, E. \& MOITA NETO, J. 2011. Logística Reversa nas Indústrias de Plásticos de Teresina-PI: Um Estudo de Viabilidade. Polimeros, 21, 246-251.

DE BRITO M. P. \& VAN DER LAAN, E. A. 2010. Supply Chain Management and Sustainability: Procrastinating Integration in Mainstream Research. Sustainability 2: 859-870.

DE BRITO, M. P. \& DEKKER, R. 2004. A Framework for Reverse Logistics. En: DEKKER, R. FLEISCHMANN, M. INDERFURTH, K. \& WASSENHOVE, L. N. V. (eds). Reverse Logistics: Quantitative Models for Closed-Loop Supply Chain, Springer-Verlag: 1-27. Berlin-Germany.

DE BRITO, M. P. 2003. Managing Reverse Logistics or Reversing Logistics Management?. En: Erasmus Research Institute of Management (ERIM). PhD Thesis Rotterdam: Erasmus University Rotterdam. 324 pp.

DE SEQUEIRA, J. E. 2009. El principio de responsabilidad de Hans Jonas. Bioethikos 3: 171-193.

DÍAZ, A. ÁLVAREZ, M. J. \& GONZÁLEZ, P. 2004. Logística inversa y medio ambiente. Mc Graw-Hill Interamericana de España. 2004. 81-109 pp.

FAJARDO, L. 2006. Desarrollo Humano Sustentable: Concepto y Naturaleza. Civilizar. Ciencias Sociales y Humanas, 10. 
GOLINSKA, P. \& ROMANO, C. A. 2012. Environmental issues in supply chain management: new trends and applications. Springer- Verlag, Berlin. 266 pp.

HERRERA, C. 2009. El sector empresarial y la contaminación urbana. Revista de Ingeniería. Universidad de los Andes 30: 151160.

LEITE, P. R. 2010. Logística Reversa: meio ambiente e competitividade 2a ed. Pearson, Sao Paulo. 250 pp.

MARTIN, M. 2001. Implementing the industrial ecology approach with reverse logistics. En: Sarkis, J. (ed). Greener Manufacturing and Operations: from design to delivery and back . 2535.Greenleaf Publishing, Sheffield, UK.

MIHI, A. 2007. Nuevos beneficios de la logística inversa para empresas europeas y colombianas. Revista Universidad \& Empresa 6: 48-61.

MONROY, N. \& AHUMADA, M. C. 2006. Logística Reversa: Retos para la Ingeniería Industrial. Revista de Ingeniería. Universidad de los Andes 23: 23-33.

MUÑOZ, M. \& MUÑOZ, D. 2007. Producción más limpia en la formulación de un plan de manejo de residuos sólidos en una planta procesadora del sector lacteo. Ciencias Agropecuarias, 5, $55-63$

PÉREZ, M. \& ROJAS, J. 2010. Marco conceptual del desarrollo sostenible. En: PÉREZ, M. ROJAS, M. \& ORDOÑEZ, C. (eds). Desarrollo Sostenible: principios, aplicaciones y lineamientos de política para Colombia. Universidad del Valle, Cali. 346 pp.

PNUMA. Programa de las Naciones Unidas para el Medio Ambiente, Oficina Regional para América Latina y el Caribe. 2012. Producción Limpia. Accesado el 22 de Mayo de 2012 http://www.pnuma.org/industria/produccion_limpia.php

ROGERS, D. S. \& TIBBEN-LEMBKE, R. S. 1998. Reverse Logistics and the Environment. En: COUNCIL, R. L. E. (ed), Going Backwards: Reverse Logistics Trends and Practices: 101136.

ROLDÁN, J. BEN-DOV, Y. \& GUERRERO, G. 2004. La complementariedad: una filosofía para el siglo XXI. Universidad del Valle, Cali. 210 pp.

SANTAMARÍA, L. 1997. El desarrollo humano sostenible en el marco de la integracion americana. Documentos Ocasionales No.44, 37, 37-61.

SILVA, T. F. 2011. Principales retos de las Mipymes de cara a la globalización. En Visión Mipyme Cali: ACOPI Valle del Cauca.

VAN HOOF, B. \& HERRERA, C. M. 2007. La evolución y el futuro de la producción más limpia en Colombia. Revista de Ingeniería, Universidad de los Andes 26: 101-120.
ZENG, S. X. LIU, H. C. TAM, C. M. \& SHAO, Y. K. 2008. Cluster analysis for studying industrial sustainability: an empirical study in Shanghai. Journal of Cleaner Production 16: 1090-1097. 